\title{
EL LÉXICO ESPAÑOL EN AÏN TEMOUCHENT
}

\author{
Faiza Mechernene \\ Universidad Abou Bakr Belkaid de Tremecén
}

\begin{abstract}
Resumen
Estudio del léxico hispánico presente en el habla de la localidad de Aïn Temouchent, población ubicada en la región occidental de Argelia. Gracias a un estudio estadístico y sociolingüístico previo, se presentan en este artículo los resultados de la investigación. Se reproduce un glosario de hispanismos léxicos propios de esta región, incluyendo cada entrada detalles y ejemplos de su uso en la variante dialectal del árabe de Aïn Temouchent.
\end{abstract}

Palabras clave: Español en Argelia, Aïn Temouchent, lexicología, hispanismo léxico.

\section{ObJeto, MEtodología y ESTRUCTURA}

En el año 2015 defendimos nuestro trabajo final del máster en lengua y comunicación, dentro de la sección de español de la facultad de letras y lenguas de la Universidad Abou Bakr Belkaid de Tremecén. El trabajo llevaba por título El léxico español en el oeste de Argelia: Ä̈n Temouchent como paradigma, y estaba dirigido por el profesor Salah Eddine Salhi. Presentamos a continuación una breve descripción de sus características, objeto y metodología, junto a las principales conclusiones de este trabajo de investigación.

El marco inicial de aproximación pretendía un enfoque sociolingüístico en el que intentábamos analizar todo hispanismo usado en el habla de la ciudad de Aïn Temouchent, ubicada en el occidente argelino. La riqueza de la situación lingüística en Argelia la convierte en una fuente fértil de investigaciones. De hecho, el marco lingüístico de Argelia ha experimentado y continúa experimentando cambios significativos como resultado de la coexistencia de múltiples idiomas y variedades dialectales. Como la historia testimonia, la geografía de la actual Argelia fue cruce de caminos desde el inicio de los tiempos, se sintió atraída por diversas civilizaciones, y la han gobernado dinastías de bereberes, árabes y turcos otomanos, además de franceses y españoles. Esta última nación es uno de 
los países cuyas tropas han permanecido más tiempo en la costa argelina, más de trescientos años, notablemente en las zonas del oeste, Orán, Aïn Temouchent, Mostaganem, Tremecén, Saïda, Sidi Belabés o Béchar.

Los habitantes del oeste de Argelia, a través de su coexistencia con el elemento español, han adoptado a lo largo de tantos años de convivencia y vecindad española una mezcla de culturas y códigos con influencia hispánica patente en su naturaleza social, cultural, gastronómica, folclórica y, por supuesto, lingüística. Esta última se manifiesta en forma de préstamos, léxicos o interferencias que tienen origen en el sistema español, y se integran en el dialecto vernáculo de Argelia, especialmente en la variante local occidental.

Durante nuestra investigación, hemos abordado el habla cotidiana de los habitantes de la llamada "perla de Orán”, la ciudad de Aïn Temouchent. Hemos intentado identificar, en la dariya o el habla dialectal que ellos emplean, los préstamos lingüísticos resultado del contacto de la lengua española con el árabe dialectal, lenguas cuyas estructuras son totalmente diferentes. Nuestro objetivo general en ese trabajo de fin de máster ha sido descubrir y construir el corpus de préstamos lingüísticos de origen español, circunscritos dentro del sistema del árabe dialectal, para dar explicación al fenómeno lingüístico del hispanismo. Así pues, hemos centrado nuestro estudio en la identificación de los hispanismos que los habitantes de Aïn Temouchent preservan en su habla.

Los objetivos específicos que nos proponemos en este trabajo implican, en primer lugar, un acercamiento al elemento histórico y sociolingüístico de la comunidad de hablantes de esta zona. En segundo lugar, es necesario recopilar el material léxico del español, sobre el que se realiza un glosario de los vocablos de origen español utilizados en esta zona, para pasar a comprobar su etimología, su definición en diccionarios españoles, con el fin de analizar todas las palabras de origen español, viendo si han mantenido el mismo sentido tan como se define los diccionarios.

A partir de los antecedentes históricos y los diferentes periodos en los que los españoles se instalaron en el oeste de Argelia, vamos a estudiar los elementos lingüísticos que presentan, a su vez, el contacto adquirido desde hace tiempo por los autóctonos y de ahí nos preguntamos: ¿En qué medida ha afectado el léxico español al habla del oeste de Argelia?, 
¿qué han preservado hasta ahora los habitantes de Ain Temouchent y sus alrededores de este legado lingüístico?, ¿qué generó ese contacto de lenguas?, ¿qué préstamos lingüísticos encontramos actualmente en esa habla? y ¿̇qué interferencias se han producido entre los dos códigos lingüísticos?

Éstas son las preguntas que animan nuestro interés en la realización de este trabajo de investigación, con el fin de lograr poner en evidencia la presencia de los hispanismos en el habla argelina, concretamente en Aïn Temouchent, y tratar de dar algunas respuestas a la problemática planteada. El interés que nos llevó a profundizar en la línea de la dialectología y la sociolingüística es el factor importante que presentan esas dos disciplinas para elaborar nuestro trabajo.

Tras muchos viajes a la mencionada ciudad para realizar encuestas y entrevistas, hemos recopilado el material léxico, con el fin de explicar los fenómenos semánticos a partir de un campo léxico sociolingüísticamente perfilado. Esta tarea se realizó con puntos de selección específicos, en los principales barrios de Aïn Temouchent, en el puerto con los pescadores de Bouzedjar, en las casas de algunas familias y algunas amigas, y en lugares públicos.

En cuanto a la división de los capítulos, el primer capítulo lo dedicamos a los antecedentes históricos de la zona oeste de Argelia. Es decir, tratamos la presencia española en el Oranesado desde principios del siglo XVI hasta finales del siglo XX, pasando a la lucha de los habitantes de la ciudad de Aïn Temouchent contra los colonos españoles, haciendo hincapié en el fenómeno de la emigración hacia Argelia, y su influencia e impacto sobre la cultura española en Argelia, concluyendo con el multiculturalismo, interculturalidad e interculturalidad. En el segundo capítulo, el cual abarca la lengua y la sociedad, tratamos, en parte, la labor teórica del campo de la sociolingüística, y luego, pasamos a describir los distintos fenómenos lingüísticos que se pueden dar en un contexto de lenguas en contacto, entre la población autóctona de las ciudades del oeste de Argelia (Ain Temouchent como paradigma), con la del extranjero o el inmigrante, tales como: el bilingüismo, la diglosia y el préstamo lingüístico. Estos fenómenos nos ayudan a justificar de forma científica los cambios que han afectado a la lengua de los autóctonos. Hacemos también una referencia sobre el significado del hispanismo, y terminamos 
este capítulo con la situación sociolingüística en Aïn Temouchent, para valorar la particular diversidad lingüística que existe en esta ciudad situada en la costa occidental de Argelia.

El tercer y último capítulo aborda la parte empírica del trabajo sociolingüístico partiendo de una muestra. Se ha necesitado distribuir los cuestionarios según el grado de escolaridad, sexo y generaciones, según la residencia, según los hablantes del español en fecha 2015, según el dominio en el que se utiliza el léxico español y, el último campo, según los usuarios del español en el dialecto vernáculo. Pasamos a dar una breve observación de la encuesta y el corpus. Entonces, constituyendo un cuestionario personal lingüístico de los informantes que contiene unas preguntas sobre los hispanismos más utilizados en el habla de los habitantes de Aï Temouchent, se interpretan las entrevistas. Finalizamos este capítulo con una interpretación de los hispanismos, con un breve estudio morfológico, fonético y semántico, en el que analizamos con profundidad la tipología de los hispanismos utilizados en las diferentes partes de la comunidad. Terminamos con un estudio sobre la toponimia hispánica en el área de estudio.

Señalamos que dentro de cada capítulo, hemos elaborado breves introducciones y breves conclusiones que resumen el desarrollo de cada uno, y constituyen un nexo entre un capítulo y otro.

Nos hemos acercado a aquellas obras que podían ofrecer una información más acorde con los objetivos propuestos. Así, para lo referente a la historia de Argelia, hemos tratado obras tales como las de Juan B. Vilar, Emigración española a Argelia (I830-I900), Los españoles en la Argelia francesa (I830-I9I4), Moreno Fernández en su artículo, "El español en el norte de África", las tesis doctorales de Ahmed Kaddour, Contribución al estudio de los hispanismos en el oeste de Argelia: corpus léxico, análisis fonético morfológico y semántico; Miloud Reguig, Ain Temouchent, a través de la edad; y Mohammed Kali, Ain Temouchent, le temps de la colonie, y para la metodología Marc Jaquemain, Méthodologie de l'enquête.

Para facilitar la parte práctica, hemos escogido también obras relacionadas con las disciplinas Sociolingüística y Dialectología. En esta línea, podemos citar lingüistas notables que se interesaron en el estudio de la presencia del léxico español en el habla de los argelinos, como Benallou 
Lamine (1992), Moussaoui Meftah Meriem (1992) y (2004), Moreno Fernández (1992), y Ahmed Kaddour (2013).

\section{Glosario de hispanismos en Aïn Temouchent}

A continuación damos una lista con los principales resultados de nuestra investigación, detallando el hispanismo, la etimología y un ejemplo en dialecto argelino donde aparezca el uso de la palabra:

\section{Abogado, da [bo'yado] n. m. yf.}

ETIM. del lat. advocātus

Por ej. "Khaṣni bogädo bāch yfrili had lproblām" "Necesito un abogado para resolver mi problema"

\section{Ajedrez [ $\left.a^{\prime} \operatorname{traj} x\right]$ n.f.}

ETIM. Del ár. hisp. aššațranğ o aššițranğ, este del ár. clás. šiṭranğ, este del pelvi čatrang, y este del sánscr. čaturanga 'de cuatro miembros'

Por ej. "Ayā mgāya nlagbou chätränj”" "Mohamed, vamos a jugar el Ajedrez"

\section{Alubia ['lußja] n. m.}

ETIM. del ár. hisp. allúbya, este del ár. clás. lúbiyā’, y este del persa lubeyā

Por ej. "Lubiya had lyamat raha ghalya" "Alubia está muy cara en estos días"

\section{Anchoa [lan'\}oa] n. f.}

ETIM. Del lat. apiuva, de aphye, y este del gr. à $\varphi v ́ \eta$

\section{Armario ['marju] n. m.}

ETIM. del lat. armarüum

Por ej. "Had Lmariu rah maamer belkach" "Este armario está lleno de ropa"

\section{Arrancar [řanek] v. intr.}

ETIM.de or. inc. Coromines lo da por derivado de una forma antigua "esrancar", a su vez del provenzal antiguo "ranc" o el francés antiguo "ranc"

Por ej. "Aya ranek aaliya" "Arranca" 
Astuto, ta [s'tut] [s'tuta] adj.
ETIM. Del lat. astūtus
Por ej. "Khouk sghir setuut" "Tu hermano pequeño es astuto"

Bacina [ba'sina] n. f.

ETIM.del lat. mediev. bacia

Por ej. "Jibli lbasina taa Ima" "Tráeme la bacina de agua"

\section{Balde [ßalde] n. m.}

ETIM. de or. inc.

Por ej. "Aatini lbalde bach njib sima" "Dame el balde para traer el cemento"

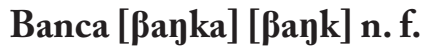

ETIM. de banco, asiento

Se utiliza para significar un asiento. Por ej. "yallab njemzou fi hada lbanc" "Vamos a sentarnos en esta banca". Y otro sentido, Por ej. "Rani rayeh lel banca" "Voy a la banca".

\section{Barco $[\beta$ arko] n. m.}

ETIM. de barca

Por ej. "Lbarco kelaa" El barco salió"

\section{Barato [ba'rato] n. m.}

ETIM. de baratar

Una palabra utilizada, cuando compran una cosa de poco precio o si encuentran el mismo tipo de algo en gran cantidad en todos mercados.

Por ej. "Rouh l'soug koulchi rah barato" "Vete al mercado todo está barato"

\section{Barra [ßařa] n.f.}

ETIM. de or. inc. quizás del latín vulgar.

Por ej. "Farid dreb sabbeh belbarra taa lehdid” "Farid golpeó su amigo con una barra de bierro"

\section{Barraca [ba'řaka] n. f. ETIM. del Cat.}


Por ej. "Hadak chibani meskin rah saken fi barraca". "Este pobre viejo vive en una barraca"

"Si Ahmed rab hal barraca ybiaa fiha dokhan" "Si Abmed se abre una barraca para vender cigarrillos"

Basta, ['basta] interj. u. tb.

ETIM. de bastar (basta, interjección procedente del imperativo de bastar)

Se utiliza para detener un escándalo, una pelea o guardar el silencio. Por ej. "Aya basta men had zega, rasi wjazni" "Basta de este alboroto, la cabeza me duele"

\section{Basura [ba'sura] n. f.}

ETIM. del lat. versūra, de verrěre, barrer

Por ej. "kis had lbasura" "Echa esta basura"

\section{Besugo [be'suyo] n. m.}

ETIM.besugo

Por ej. "Ma seydnach bzf lbesugo" No hemos pescado mucho de besugo"

\section{Betún ['biton] n. m.}

ESTIM. del lat. Bitumen

Es una mezcla de cemento y al agua, se utiliza para la construcción de casas. "Lbiton marahch mkhdoum mlih" "El betún no está bien preparado"

\section{Bobina [bu'ßina] n. f.}

ETIM. del fr. "Bobine"

Por ej. "Jibili lbobina ntaa lkhayt bach nkbayet had jipa" "Tráeme la bobina de hilo para coser esta falda"

\section{Bola ['bola] n. f.}

ETIM. del prov. bola, y este del lat. bŭlla, burbuja, bola

Esta palabra se utiliza de otro sentido para expresar el futbol. Por ej. "Wled jarna khervenli lbola taai" "Wl hijo de nos vecino me robo el balón" 
También "Lyoum nlaabou lbola maa wlad boujemaa" "Hoy, vamos a jugar balón o futbol con wlad boujmaa"

\section{Bomba ['bumba] n. f.}

ETIM. de bombo, y este del latín medieval "bombus" ("ruido"), en los clásicos "zumbido", del griego antiguo "ßó $\mu \beta \varsigma$ ” (bómbos), probablemente de origen onomatopéyico.

Por ej. "Smaatou belboumba li darouba fi felistine" "Han escuchado de la bomba que....." O otro sentido cuando alguien miente o pelea; por ej. "Malah hadek yboumbé aalina"'Porque miente esta persona"

\section{Bolsa ['bursa] n. f.}

ETIM. del lat. "bursa"

Por ej. "Aatini bursa nhout fiha had swalab" "Dame una bolsa para poner esta compra"

\section{Borracho [ba'rat $\left.\int o\right]$ adj. m. us. en desus.}

ETIM. del Cat. morratxa ("redoma")

Ebrio, embriagado por la bebida. El empapado en almíbar y vino generoso.

Por ej. "Didi barracho", Didi es un personaje de la película argelina Al-Harik, una persona intoxicada por el alcohol. Hasta la actualidad los niños del oeste de Argelia utilizan esta frase cuando juegan entre ellos "Didiii barrachooo, didiii barracho..."

\section{Borrico [bo'řiko] adj. m.}

ETIM. del lat. tardío burrīcus 'caballo pequeño

Se usa por el alumno que no estudia bien, por ej. "Borrico la clase" "Esta el borrico de la clase"

\section{Bota ['bota] n. f.}

ETIM. del fr. botte

Por ej. "Aandek lbota”" ¿Tienes botas?" 


\section{Bravo, [bravo] adj. Colq.}

ETIM. del lat. pravus, malo, inculto

Se utiliza cuando alguien hace una cosa de bueno o gana en un juego, en el trabajo o en sus estudios.

Por ej. "Bravooo!"

\section{Cacerola [kas'řona] n.f.}

ETIM. de cazo

Por ej. "AAtini casrona taa lhelib" "Dame cacerola de leche"

Cabeza [ka’ßesa] n. f. us. pop.

ETIM. del castellano antiguo "cabeça" y está bajo del lat. "Capitĩa".

Por ej: "Lcabesa andek habsa" "Tu cabeza no funciona"

Cable, ['kaßle] n. m.

ETIM. de or. inc.; quizá del b. lat. "Capŭlum", "cuerda"

Por ej. "Rani nsegued lcable taa tricite" "Reparo el cable de la electricidad"

Cabrero [ka'ßrero] n. m. y f.

ETIM. del lat. caprarĭus

Calabaza [calbosa] n. f.

ETIM. de calabaza

Por ej. "Hadi chira calbosaaa!" Esta chiquilla es como una calabaza"

Calamar [kala'mar] n.f.

ETIM. del lat. calamarǔus, de calămus, caña o pluma de escribir

Caldero [kar`ero] n. m.

ETIM. del lat. caldarĭum

Un plato que se prepara por parte de pescadores

Caldo ['kaldo] n. m.

ETIM. del lat. caldus, caliente 


\section{Calesa [ka'lif] n.f.}

ETIM. del fr. calèche, y este del checo kolesa

Por ej. "Aami Ahmed aandeh lkalish chebab" "Tio Ahmed tiene una calesa hermosa"

\section{Caliente [karaN 'tita] ['karan] ['lami] adj.}

ETIM. del lat. calens, -entis

Por ej. "Aatini mil cinq thon karantita" "Dame un mil cinco de caliente con atún"

\section{Calma ['kalma]n. f. calmar [t'kalma] v. intr.

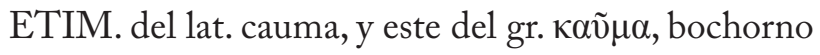 \\ Por ej. "Lcalma raha mliha" "El clima está bien"}

\section{Calzón ['kalso] n. m.}

ETIM. de calzón

Por ej: "Lbesi lweldek calson" "Pones a tu niño un calzón"

\section{Cantina [kaN'tina] n. f.}

ETIM. del it. Cantina

Puesto público en que se venden bebidas y algunos comestibles. Sótano donde se guarda el vino para el consumo de la casa. Pieza de la casa donde se tiene el repuesto del agua para beber.

\section{Capazo [ka'ßaso] n. m.}

ETIM. del lat. "capax, -ācis, capaz"

Se utiliza para la compra. Por ej. "Ma rani rayeh lelmarché aatini lcabaso" "Madre mía, me voy al mercado, dame el capazo"

\section{Carreta [ka'řita] n. f.}

ETIM.del dim. (-eta) de carro y este del lat. carrus

Dicho de un mal coche. Por ej. "Nta aandek carrita machi loto" "Tienes una carreta" 


\section{Carro ['kařo] ] n. m.}

ETIM. del lat. carrus, y este del galo carros

Por ej. "Jeddek ybiaa lkhodra fi carro?” “¿Vende tu abuelo legumbres en un carro?"

\section{Carroza [ka'řosa] n. f.}

ETIM. -del lat. Carrūca

Por ej. "Hadik chira aandha carrosa hamra" "Esta chica tiene una carroza roja"

\section{Carta ['karta] n. f.}

ETIM. del lat. charta, y este del gr. $\chi \alpha$ ó

Por ej. "Aya sabbi rwah nlaabou lcarta" Amigo vamos a jugar la carta"

También "Jibili benti lcarta taai bach nkblous" "Mi niña, tráeme mi tarjeta de identidad para el pago"

\section{Cartagena [karta 'xena] n. pro. topónim.}

Se utiliza para significar un viaje muy largo, o un lugar remoto.

Por ej. "Rah hatta lcartagena w ja" "Se fue hasta Cartagena y volvio"

\section{Chamanto [ ar'mato] n. m.}

ETIM. chalmate: quizá del náhuatl chalani, part. de ochalan, romperse, $y$ matate, red

Un objeto de tela o lana fina que sirve para cubrirse.

Por ej. "Lberd rah lyoum, ghetini bcharmato" "Hoy hace frio, cúbreme de chamanto"

Chamar [Ja'mer] v. tr.

ETIM. disc. cf. port. 'cambar', 'cambiar', fr. 'changer'

Por ej. "Aya chammer ala yeddik w khedem" "Chama y trabaja"

\section{Chumbo ['t $\int$ ambo] $n$. m.}

ETIM. del port. Chumbo (plomo) Y este del lat. plumbum. Higo chumbo.

Por ej. "Aatini chuia chambo" "Dame unos cuantos chumbos" 
84 Faiza Mechernene

Chancla ['Jangla] n. f.

ETIM. cf, chanca

Por ej. "Jibili changla" "Tráeme mi chancla"

\section{Chancleta [ ag'gleta] n. f.}

ETIM. Del dim. de chancla

Chinela sin talón, o chinela o zapato con el talón doblado, que suele usarse dentro de casa.

\section{Chaqueta [xa'kita] n. f.}

ETIM. de jaqueta

Por ej. "Hadi jaquita hamiya" "Es un chaqueta cálida"

\section{Cigarro ['gařo]}

ETIM. del maya siyar

Por ej. "A sabbi aatini garro" "Amigo mío dame un cigarro"

\section{Cintura [0eN'tura] n.f.}

ETIM. del lat. cinctūra

Parte de una prenda de vestir que corresponde a esta parte del cuerpo.

\section{Chicha ['t $\left.\int \mathrm{i} \int \mathrm{a}\right] \mathrm{n} . \mathrm{f}$.}

ETIM. de la voz aborigen del Panamá chichab, maíz

Por ej. "Emmmm! Tchicha harra" "Emm! ¡Qué buena chicha!"

\section{Cisterna [0i'tirna] n. f.}

ETIM. del lat. cisterna

Por ej. "Weldi aamer citirna raha kbawya" "Hijo, la cisterna está vacia, llénala"

\section{Cochura ['kufa] n. f.}

ETIM. del lat. coctūra

Por ej. "Lkucha taa jaretna tyeb ghaya lkbobz" "Chritlkoum lyoum croissant mel kucha" 


\section{Cocina [ko' $\theta$ ina] n. f.}

ETIM. del lat. coquīna, de coquĕre, cocer

Por ej. "Ya weldi jibli lma mel cosina" "Mi niño tráeme un vaso de agua de la cocina"

Cola ['kola] n.f.

ETIM. del lat. colla, y este del gr. кó $\lambda \lambda \alpha$

Por ej. "Lyoum lcola raha fi lbushta" "Hoy hay mucha gente en la oficina de correos"

Comisaría [komi'sarja] n. m. y f.

ETIM. del bajo lat. commissarǔus, y este del lat. commissus, part. pas. de committěre, cometer.

Por ej. "Andi aami yekbdem fi lcomisaria" "Tengo un tío que trabaja en la comisaria”

\section{Contra ['koNtra] prep. \\ ETIM. del lat. "contra"}

\section{Contrabando [tra' $\beta$ aNdo] n. m.}

ETIM. de contra y bando, edicto, ley

Por ej. "Hada chir ykbdem trabando" "Este chico trabaja de contrabando"

Corona ['grona] $\mathbf{n . f}$

ETIM. del lat. "Corōna"

Costa ['kosta] n. f.

ETIM. del gall. o cat. "Costa"

Cubierta [ku'Birta] n.f.

ETIM. de cubierto

Por ej. "Ghatini bel cubirta ysejik" "Cúbreme”

Dama ['dama] n.f.

ETIM. del fr. dame, y este del lat. dominna

Por ej. "Taaref lcarta taa damma?” "CConoces el juego de cartas de Dama?” 


\section{Dominó [do'mino] n. m.}

ETIM. del fr. domino, y este del m. or. que dómino

Por ej. "Rah aandi domino fi dar aya rwah tlaab maaya" "Vete conmigo para jugar al domino"

\section{Escalera [ska'lera] n.f.}

ETIM. del lat. "scalarīa", pl. n. de "scalāres"

Por ej. "Scalera taa lbatimat aayatni" "Las escaleras de los edificios me cansan"

\section{Escuela [sa'kwela] n. f.}

ETIM. del lat. "schola”, y este del gr. " $\sigma \chi 0 \lambda \eta ́ "$

Se utiliza para la escuela primaria.

Por ej. "Rouh jib khouk m sakuela" "Traerá tu hermano de la escuela"

\section{España [z’ßanja] n. pro.}

ETIM. España

Por ej. "rani rayeh lsbania" "Voy a España"

\section{Fábrica [fa'ßrika] n. f.}

ETIM. del lat. 'fabrǐca'

\section{Factura [fak'tura] n. f.}

ETIM. del lat. factūra

Por ej. "Wsletni lyoum lfactura taa Ima" "Hoy he recibido la factura de agua"

\section{Falta [falta] n. f.}

ETIM. del lat. vulg. "fallĭta"

Se utiliza cuando alguien hace un error o una falta.

Por ej. "Sabebti lyom daret wahed lfaltaaa" "Mi amiga ha hecho una falta"

\section{Falsa ['falsa] adj. f.}

ETIM. del lat. "falsus"

Se utiliza por parte de gente mayor (jas) cuando uno de sus niños 
hace un gran error.

Por ej. "Yaaaw derti falsa gued rasek" "Has hecho una gran falsa"

Familia [fa'milja] n.f.

ETIM. del lat. "familia"

Por ej. "Lfamilia raha ghaya?” ¿Está bien la familia?”

\section{Farda [f'rða] n. f.}

ETIM. disc.

Bulto o lío de ropa. Pero en el habla de los habitantes de Aïn Temouchent significa lo contrario, como muletilla para expresar nada.

Por ej. "Win raha lferda taa sebati" "Dónde está.... de mis zapatos"

Fiesta [fi' ta] n.f.

ETIM. del lat. "festa", pl. de "festum"

Por ej. "Lyoum lfhishta hatta wahed ma ykhdem" "Hoy la fiesta nadie trabaja"

Fideos [fda'we $]$ n. m. pl.

ETIM. quizá del mozár. y ár. hisp. fidáwš

Pasta alimenticia de harina en forma de cuerda delgada

Por ej. "Dertou lyoum fdawesh lelftour?" "Han preparado los fideos por la cena?"

\section{Flecha ['flit $\left.\int a\right]$ n.f.}

ETIM. del fr. flèche, y este quizá del franco *fleuk[k]a; cf. neerl. medio vlieke, b. al. ant. fliuca

Por ej. "Taaref tlaab jeu taz fletsha" "Sabes tú jugar el juego de flecha?"

Fondo ['foNdo] n. m.

ETIM. del lat. fundus

Forma ['forma] n. f.

ETIM. del lat. forma

Por ej. "Chouf lforma taa hadou scalerat chebba" "Mira estas escaleras tienen una buena forma" 
Francia ['fransa] n. pro.

ETIM. de Francia, viene del germánico frank, libre

Por ej. "Rani rayeh l'Fransa" "Me voy a Francia"

\section{Freno ['freno] n. m.}

ETIM. del lat. "frēnum"

Se utiliza para el freno del coche. O para una persona que habla mucho.

Por ej. "Lfreno taa loto rah aayan"“El freno de esta coche es malo" "Aya frini chuia rak msbah w nta tahder" "Para de hablar un poco"

\section{Gabardina [gaßar’ðina] n. f.}

ETIM. Cruce de gabán y tabardina, der. de tabardo.

\section{Gamba ['gamba] n.f.}

ETIM. del it. gamba, pierna, este del lat. vulg. camba, pierna de las caballerías, y este del gr. $\alpha \mu \pi \eta ́$, curvatura

Por ej. "Lyoum lgamba jat sghira" "La gamba de hoy es muy pequeña"

\section{Gamella [ga'mila] n. f.}

ETIM. del lat. camella, escudilla

Artesa que sirve para dar de comer y beber a los animales, fregar, lavar, etc.

Por ej. "Had lgamila chebba bchezal chritiha?" "Es una buena gamella! ¿Cuánto cuesta?"

\section{Garbanzo [gor'ßaN日o] n. m.}

ETIM. de or. inc.

Por ej. "Emmm! Ma lyoum raha mtyeba lgorbanzo" "Emmm! Mi madre ha preparado deliciosos garbanzos"

\section{Gato ['gat] n. m.}

ETIM.del lat. cattus

Por ej. "Lgat taai twedrli lbareh" "Ayer he perdido mi gato" 
Gitana [xi'tana] [am'řya] adj. m. y f.

ETIM. de egiptano, porque se creyó que procedían de Egipto

Se utiliza para expresar una mujer de mal vestido.

Por ej. "Had lmera ki lgitanat" "Esta mujer es como las gitanas"

Gorda ['gorða] adj. f.

ETIM.del lat. gurdus, voz de or. hisp.

Muy abultado y corpulento.

Grelo ['grelo] n. m.

ETIM. del lat. gryllus

Insecto ortóptero, grillo.

Por ej. "Rah kayen grelo henaya, lazem nketloub" "Hay un grelo aqui, debemos matarlo"

Grúa ['grua] n.f.

ETIM. del lat. grus, gruis

Guerra ['giřa] n. m.

ETIM. del germ. werra, pelea, discordia; cf. a. al. ant. wërra, neerl. medio warre

Guitarra [gi'tara] n. f.

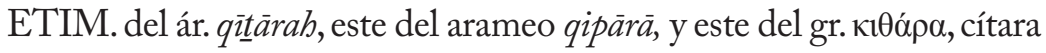

Por ej. "Hadi lguitara jabouhali cadeau" "Esa guitarra es un regalo de mi amigo"

Gurbio ['gur $\beta i]$ n. m.

ETIM. de gubia

Dicho de un instrumento de metal, que tiene alguna curvatura

Por ej. "Sabbi rah karifi wahed lgurbi allab la ycherwfek" "Mi amigo está alquilando un gurbio muy malo"

Gusto ['gusto] n. m.

ETIM. del lat. gustus 
90 Faiza Mechernene

Por ej. "Aandi lgusto fi bola" "Me gusta el futbol"

\section{Harina [fa'rina] n. f.}

ETIM. del lat. farīna

Por ej. "Khaseni lfarina bach ndir lherira" "Necesito la harina para preparar la sopa"

\section{Horno ['forno] n. m.}

ETIM. del lat. furnus

Por ej. "Teyebi lkhobz fi lforno" "Se ha preparado el pan en el horno"

\section{Hospital [zßi'tar] n. m.}

ETIM.del lat. hospitālis

\section{Japuta [xa'puta] n.f.}

ETIM. del ár. hisp. *̌abbuita, este del ár. clás. šab[b]ưt, y este del arameo šabbūtāa)

Pez teleósteo del suborden de los Acantopterigios, de color plomizo.

\section{Joder [xowa'r] interj. u.}

ETIM. de joder

Para expresar enfado, irritación, asombro, etc.

Por ej; "Hadek andeb Mercedes, jouar" "Joder, esta persona tiene Mercedes"

\section{Jurel [xu'rel] n. m.}

ETIM. del ár. hisp. šuríl, este dim. del lat. saurus, y este del gr. $\sigma \alpha \tilde{\rho} \rho \varsigma_{\text {, }}$ lagarto

Pez teleósteo marino, del suborden de los Acantopterigios.

\section{Kilo ['kilo] n. m.}

ETIM. kilo, De kilo- y gramo

Por ej. "Wzenli zouj kilo tomatis" "Pésame un kilo de Tomate"

Lámpara ['lamba] n.f.

ETIM. de lámpada 
Por ej. "Lamba taa sala nharguet" "La lámpara de la sala no funciona"

Largo(a) ['laryo] ['larya] adj. us. fig.

ETIM. del lat. largus

Por ej. "Hadek largo" "Este chico es largo"

Lejía ['lexja] n. f.

ETIM. del lat. [aqua] lixīva

Por ej. "Hadi lejia gataa" "Esta lejía es muy buena"

\section{Lista ['lista] n. m.}

ETIM. del germ. *î̂sta; cf. a. al. ant. y nórd. lîsta, ingl. list, franja, orillo

Por ej. "Lyoum afichaw lista taa lbac" "Hoy han enunciado la lista de bachillerato"

Maestro [ma'estro] adj. m.

ETIM. del lat. magister, -tri

\section{Marca ['marka] n. f.}

ETIM. del b. lat. marca, y este del germ. ${ }^{*}$ mark, territorio fronterizo; cf. nórd. mark, a. al. ant. Marka

Por ej. "Cha heya lmarca lmliba fi sechoir" "Cuál es la marca del secador" También "L'equipe marcaaat buuut" "El equipo ha marcado"

\section{Marina [ma'rina] n. pro. f.}

ETIM. del lat. marīnus

Por ej. "Jarena yekbdem filmarina" "Nuestro vecino trabaja en la marina"

\section{Marmita [mar'fita] n.f.}

ETIM. del fr. marmite

Por ej. "Lhrira raha fi lmarmita" "La sopa está en la marmita"

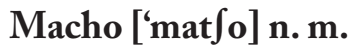

ETIM. del lat. Mascŭlus 


\section{Madrid [ma’rid] n. pro.}

Topónimo de una playa situada en Beni Saf.

\section{Miseria [mi'日irja] n. f.}

ETIM. del lat. miserǐa

Por ej. "Rana aaychin fi misiria" "Vivimos en la miseria"

\section{Motor [mo'tor] n. m.}

Por ej. "Aandi motor Yamaha" "Tengo una motocicleta Yamaha"

\section{Muca [muca] n. f.}

ETIM. del quechua muka

Para describir una mala persona.

\section{Negro ['niyru] adj.}

ETIM. del lat. niger, nigri

Por ej. "Wash nigru kach khedma" "Eh negro jestás trabajando?"

\section{Paella [pa'ela] n. f.}

ETIM. del valenciano paella

Plato de arroz seco, con carne, pescado, mariscos, legumbres, etc.

Por ej. "Chrit gaa nouaa lhout bach ntyeb lpaella" "He comprado todos tipo de pescados para preparar la paella"

\section{Pala ['bala] n. f.}

ETIM. del lat. pala

Por ej. "Aatini lbala nlem had trab" "Dame la pala para cubrir ese suelo"

\section{Paloma [ba'loma] n. f.}

ETIM. del lat. vulg. Palumba

Se utiliza de manera irónica.

Por ej. "Jaretna dayera ki paloma" "Nuestra vecina esta como una paloma"

\section{Pareja [pa'rexa] n. f.}

ETIM. del lat. paricŭlus, dim. de par, paris, igual 
Por ej. "Lbarcos mchaw pareja" "Los dos barcos se han ido en parejas"

\section{Pasarela [pas’̌rela] n. f.}

ETIM. Del it. Passerella

Se utiliza en la marina entre los pescados.

\section{Patata [ba'tata] n.f.}

ETIM. Cruce de papa y batata

Por ej. "Lbatata raha ghlya bzaf had lyamet" "La patata está muy costosa estas días"

\section{Piedra ['peðra] n.f.}

ETIM. del lat. petra

Por ej. "Nroub l'pedra lbayeda nreyah chuia" "Voy a blanca piedra para relajarme"

\section{Pintura [bN 'tura $]$ n. f. benter [bNt'r $]$ v. tr.}

ETIM. del verbo pintar

Por ej. "Ah! Hadi bentura chebba" "Esta es una hermosa pintura"

\section{Placa ['plaka] n.f.}

ETIM. del fr. plaque

Por ej. "Marakch tchouffi Iplaca taa stop" "No miras la placa de Stop"

\section{Placeta [pla'日eta] n. pro. f. cat.}

ETIM. del cat.

Es una plaza situada en el centro de la ciudad Aïn Temouchent

Por ej. "Jeddi rah gaaed maa shabah fi lplaceta" "Mi abuelo esta con sus amigos en la placeta”

\section{Plan ['blan] n. m.}

ETIM. del plano

Por ej. "Gouli waktach twejdli lblan taa dar?" "Dime ¿̨cuándo será preparado el plan de mi casa?" 


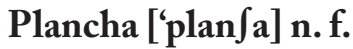

ETIM. del fr. planche

Lámina o pedazo de metal llano y delgado.

\section{Plomo ['plomo] n. m.}

ETIM. del lat. plumbum, voz de or. hisp.

\section{Policía [bu'lisja] n. f.}

ETIM. del lat. politīa, y este del gr. $\pi$ o $\lambda \iota \tau \varepsilon i ́ \alpha$

\section{Pomada [po'maða] n.f.}

ETIM. de poma

Por ej. "Tbib atani pomada taa romatiz" "El doctor me ha preescrito una pomada" También de manera vulgar, "WahTelili lpomada bech ncherilek sbat" "No me das pomada para comprarte un zapato"

\section{Popa ['popa] n. m}

ETIM. del lat. puppis, con la (a) de prora

Parte posterior de una embarcación.

\section{Poncho ['pon $\left.\int o\right]$ n. m.}

ETIM. Poncho

Por ej. "Lponcho taak chbab" "Tiene un hermoso poncho"

\section{Punto ['buNtu] n.m.}

ETIM. del lat. "punctum"

Por ej. "Ooooob bunto buntuu!» "oooh! Goool”

$\mathrm{O}$ de otra manera, por ej. "A sabbi aatini bunto taa doukban" "Amigo mio dame un cigarrillo"

\section{Ramita rama [ř́mita] n. f. bot.}

ETIM. de ramo

Por ej. "Manich aaref ramita taaek mnin" "No sé de qué ramita (clase) eres" 


\section{Remolque [ř́e'morka] n. m.}

ETIM. del v. tr. remolcar, del lat. remulcāre, y este del gr. de $\dot{\rho} \tilde{v} \mu \alpha$, cuerda, y ò $\lambda \kappa o ́ s$, tracción

Por ej. "Remorca taa l'ma" "Remolque de agua"

Río Salado n. pro. ['̌̌jo] sus. m. [sa'laðo] adj. m.

ETIM. del lat. rius, rivus, arroyo _ salado, del part. de salar

Topónimo de un municipio situado en la región de Aïn Temouchent.

\section{Roca ['̌roka] n.f.}

ETIM. de or. inc.

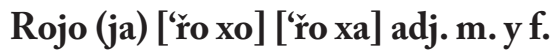

ETIM. del lat. russus

De color rubio. Dicho especialmente del cabello.

Por ej. "Hadek rojo ytchebah ghi lmab" "Este chico rubio se ve como su madre"

\section{Ronda [‘roNda] n.f.}

ETIM. voz de or. rom.

En varios juegos de naipes, vuelta o suerte de todos los jugadores. Conjunto de las tres cartas primeras que en el juego del sacanete se ofrecen a los que van a parar.

\section{Sala ['sala] n.f.}

ETIM. del germ. sal, edificio de una sola pieza de recepción.

Habitación principal de la casa.

Por ej. "Sabbeti andha laares fi sala taa al-Andalus" "Mi amiga va a hacer su matrimonio en la sala al-Andalus"

\section{Sandalia [saN'dala] n. f.}

ETIM. del lat. sandalĭum, y este del gr. $\sigma \alpha v \delta \alpha ́ \lambda ı v$

Zapato ligero y muy abierto, usado en tiempo de calor.

Por ej. "Lhoman rah, nroub nekra bsandalti" "Hace mucho calor, me voy con sandalias" 


\section{Sardina [sarðina] n.f.}

ETIM. del lat. sardīna

Por ej. "Ma syednach bzaf sardina" "No hemos pescado mucha sardina"

\section{Semana [si'mana] n. f.}

ETIM. del lat. septimāna

Por ej. "Chehal ghaya, simana jaya ma nkrawch” "Qué bueno! la semana que viene no estudiamos"

\section{Servita [š̌’bita] n.f.}

ETIM. del lat. servi

Significa la toalla para la cara.

\section{Sombrero [som'brero] n. m.}

ETIM. de sombra

Po ej. "Dir sombrero aala chems" "Este sombrero es para el sol"

\section{Sopera [su'ßera] n. f.}

ETIM. de sopa

Vasija honda en que se sirve la sopa en la mesa.

Por ej. "Win raha subera taa chourba?" ¿Dónde está la sopera de sopa?"

\section{Sota ['sota] n. f}

ETIM. del lat. subtus, debajo

La persona que dice disparates.

Por ej. "Lemra sotat" "La mujer está loca"

\section{Suma ['suma] n. f.}

ETIM. del lat. summa

Operación de sumar.

Por ej. "Chehal suma taa had sebbat?" "Cuál es la suma de este zapato?"

\section{Tabla ['taßla] n.f.}

ETIM. del lat. tabŭla

Pieza de madera plana, de poco grueso y cuyas dos caras son paralelas 
entre sí. Pieza plana y de poco espesor de alguna otra materia rígida.

Por ej. "Houti Imakla fog tabla" "Pone la comida sobre la tabla"

\section{Tambor [tambor] n. m.}

ETIM. Quizá del ár. hisp. țabbūl, hipocorístico del țabál

Instrumento musical de percusión, de madera o metal, de forma cilíndrica, hueco, cubierto por sus dos bases con piel estirada, que se toca con dos palillos.

\section{Tarta ['tarta] n.f.}

ETIM. del fr. tarte

Pastel grande, de forma generalmente redonda, relleno de frutas, crema, etc., o bien de bizcocho, pasta de almendra y otras clases de masa homogénea.

Por ej. "Klit wahed tarta taa chicula! Rawaa!" "He comido una tarta deliciosa, tarta de chocolate"

\section{Tenedor / Garfo ['garfo] [far'tita] n. m.}

ETIM. del por.

Por ej. "Kouli slata bel garfo" "Come la ensalada con el tenedor"

\section{Terco [t'ryo] adj.}

ETIM. de or. inc.

Pertinaz, obstinado e irreducible.

Por ej. "Dayera ki tergo" $\mu$ "Eres muy terco"

\section{Tisana [ti'sana] n. f.}

ETIM. Del lat. ptisāna, y este del gr. $\pi \tau \iota \sigma \alpha ́ v \eta$

Bebida medicinal que resulta del cocimiento ligero de una o varias hierbas y otros ingredientes en agua.

Por ej. "Rani m'grippé nroub nchroub tisana" "Tengo gripe, voy a tomar tisana"

\section{Tomate [to'matis] n. m.}

ETIM. del nahua tomatl 
Por ej. "Jibli zouj kilo tomatis" "Cómprame dos kilos de tomates"

$\mathrm{O}$ de us. fig. por ej. "Wjhek rah comma tomatis", "Tu cara está como un tomate"

\section{Toro ['toro] n. m.}

ETIM. Del lat. taurus

\section{Tractor [trak'tur] n. m.}

ETIM. del lat. tractus, de trahĕre, arrastrar

\section{Triunfo ['truMfu] n. m.}

ETIM. del lat. triumphus

En ciertos juegos de naipes, carta del palo de más valor.

Un juego que se juega en Aïn Temouchent y Beni Saf desde la presencia española.

\section{Vuelta [we'rta] n. f.}

ETIM. del lat. volǔta, por volūta

Por ej. "Aya ndirou werta" "Vamos a dar una vuelta por aqui"

\section{Zanahoria [0’ř̀ja] n. m.}

ETIM. del ár. hisp. safunnárya, y este del gr. $\sigma \tau \alpha \varphi v \lambda i ́ v \eta \alpha ̉ \gamma \rho i ́ \alpha$, zanahoria silvestre

\section{Zapato [z'ßat] n. m.}

ETIM. del turco zabata

Por ej. "Papa aatini necheri sebat jdid" "Papa me ha dado dinero para comprar unos zapatos nuevos"

\section{Conclusiones}

La conclusión principal de nuestro trabajo es que, hasta hoy día, los nativos de Aïn Temouchent, mayores y menores, preservan un léxico español que se presenta como contacto lingüístico entre el árabe dialectal y la lengua española en forma de interferencias o préstamos entre ambos códigos. 
Hemos recopilado más de 150 hispanismos, corpus en el que hemos descubierto interferencias entre el árabe vernáculo y el español como en los casos de zroudia-zanahoria, tomatis-tomates, charmato-calmante, remorca-remolque. Encontramos otras interferencias un poco más complejas, que se clasifican como galicismos parciales, es decir, una palabra procedente al mismo tiempo de dos lenguas y que se usa en el habla de esa zona occidental, quizás para facilitar su habla. Citamos como ejemplos la palabra vista, que toma el significado de la palabra francesa veste, y el significante de la palabra española vista. También es el mismo caso de las palabras tablall, table-tabla, firmall-ferme, firma, blouzall blouse, blusa, sabon\| savon, jabón. Constatamos finalmente la presencia del préstamo lingüístico sin alterar en casos como Madrid, placeta, chumbo, macho, etc.

Como se puede notar, el presente estudio contiene una parte teórico y una práctica. En la parte teórica, hemos realizado una lectura detallada de los antecedentes históricos de Argelia, en la que hemos resaltado y justificado las más importantes causas y consecuencias de este legado extranjero lingüístico y cultural, que existe en este país de África del norte. En base a opiniones y citas de diferentes lingüistas, hemos dado definiciones a algunos conceptos claves relativos a nuestro trabajo, tratando el contacto de lenguas, el bilingüismo, la diglosia, el multiculturalismo, interculturalismo y la interculturalidad, pasando a las actitudes y representaciones sociolingüísticas, dando una presentación acerca de la situación sociolingüística de Aïn Temouchent. Al final hemos identificado la complejidad lingüística que rodea a los habitantes de esta región. Este censo nos ha llevado a construir un marco para comprender mejor nuestra problemática. En la parte sociolingüística y gracias a las encuestas que hemos practicado, se concluye que la generación de más de 65 años utiliza mucho el elemento español en su dialecto, debido a su convivencia con los españoles antes y durante la colonización francesa. Por eso, hemos notado que hay algunos hispanismos que se encuentran en desaparición ya que no son utilizados por parte de los jóvenes que usan un árabe vernáculo mezclado con francés más que con español. Pero no se puede negar que la generación que surge usa el elemento español de manera académica, es decir, en las escuelas, institutos o durante sus viajes a España debido a un clima de mayor relación en las últimas décadas entre las dos orillas. 
Desde el corpus recopilado, confirmamos que la gente de esta región conserva un legado lingüístico cultural que se usa en diversas aéreas: la marina (herramientas navales, nombres de peces; pasarella, calma, sardina, calamar, barco, khouril...), la casa (cocina, armario, lekhia,...), la calle (arrancar, khouar...), la gastronomía y alimentación (tarta, chocolate, tomatis, zroudia, paella, chumbo, chicha, tomatis,...), los juegos (triunfo, dama,...), vestidos y calzados (sebbat, ropa, chancla, chancleta...), la toponimia (Madrid, río salado), la música (pandero, guitara,...), objetos de agricultura y albañilería (remorca, citerna, balde, maseta, masa,...), medios de transporte (carrosa, carreta, carro,...), la fauna y la flora (mancho, rasa, ramita, moca,...).

En este trabajo de investigación hemos señalado que el estudio morfológico nos orienta a poner cada hispanismo en su categoría morfológica. También hemos encontrado cambios o modificaciones y sustituciones entre las vocales y consonantes citadas en la parte del estudio fonético. En el estudio semántico se ofrece la tipología de los hispanismos, en el que hemos clasificado cada hispanismo dentro de su significado usado por parte de la población del occidente de Argelia. Y en el estudio de onomástica se ha estudiado el origen y significado de cada nombre propio.

Concluimos que la región de Ain Temouchent disfruta de una riqueza lingüística que ocupa una parte estratégica y significativa dentro de la historia cultural del occidente de Argelia. Esos elementos extranjeros participan actualmente en Aïn Temouchent en los ámbitos de la vida diaria agrícola, marítima y cotidiana, como parte consustancial del habla y la cultura locales ${ }^{1}$.

1 Texto completo de la tesis aquí resumida puede encontrarse en el siguiente enlace: http://argelina.org/wp-content/uploads/TFM-el-lexico-espanol-en-el-oeste-de-argelia.pdf 